\title{
MONOTONIC SUBSEQUENCES
}

\author{
JOSEPH B. KRUSKAL, JR.
}

1. Introduction. Hidden in a paper by Erdös and Szekeres ${ }^{1}$ is an intriguing result.

BASIC Theorem. Every sequence $S=\left\{x_{i}\right\}\left(i=1\right.$ to $\left.n^{2}+1\right)$ of real numbers having $\left(n^{2}+1\right)$ terms possesses a (perhaps not strictly) monotonic subsequence $M=\left\{x_{i_{j}}\right\} \quad(j=1$ to $n+1)$ having $(n+1)$ terms. Furthermore $\left(n^{2}+1\right)$ is the smallest number for which this is true.

Briefly, this theorem states that a monotonic subsequence of any desired length can be picked out from a sufficiently long sequence, and gives the precise lengths. An elegant proof of this theorem (unpublished) which is due to Martin D. Kruskal is sketched here.

Notation. Let $S$ and $T$ denote sequences, and let $M$ and $N$ denote monotonic sequences. Let $S(p)$, etc., denote a sequence having $p$ terms. Let $\psi(n)$ denote the least integer $p$ such that every $S(p)$ contains an $M(n)$. In this notation we may restate the basic theorem thus:

BASIC TheOREM. For sequences of real numbers, $\psi(n+1)=n^{2}+1$.

To show that $\psi(n+1) \geqq n^{2}+1$, it is sufficient to exhibit an $S\left(n^{2}\right)$ which contains no $M(n+1)$. Such a sequence is the following:

$$
n, \cdots, 1,2 n, \cdots, n+1, \cdots, n^{2}, \cdots, n^{2}-n+1 \text {. }
$$

To show that $\psi(n+1) \leqq n^{2}+1$, assume the contrary and let $n$ be the least integer such that $\psi(n+1)>n^{2}+1$. Let $S\left(n^{2}+1\right)$ be a sequence which does not contain any $M(n+1)$. Now define a majorant (minor$a n t$ ) of $S$ to be a term which is strictly greater (smaller) than all terms following it in $S$. The majorants (minorants) form a decreasing (increasing) subsequence of $S$; hence there are at most $n$ majorants and $n$ minorants. As the final term of $S$ is necessarily both a majorant and a minorant, there are at most $(2 n-1)$ extremants (majorants and minorants). The last term of every $M(n)$ contained in $S$ must be an extremant. Now delete from $S$ all its extremants. The remainder $S^{\prime}$ can contain no $M(n)$, yet has at least $\left[\left(n^{2}+1\right)-(2 n-1)\right]=\left[(n-1)^{2}\right.$ +1 ] terms. Hence $\psi(n) \equiv \psi(n-1+1)>(n-1)^{2}+1$. This contradicts the definition of $n$ and completes the proof.

Received by the editors April 7, 1952.

1 P. Erdös and G. Szekeres, A combinatorial theorem in geometry, Compositio Mathematica vol. 2 (1935) p. 463 . See the theorem on p. 467. 
2. First generalization-real vector spaces. In the following two sections the concept of a monotonic sequence is generalized to sequences of vectors from a finite-dimensional real vector space and a partial analogue of the Basic Theorem is obtained. (If $S=\left\{x_{i}\right\}$ is a sequence from a vector space, the subscript still distinguishes terms of the sequence, not components of a vector.)

Note that a sequence $S=\left\{x_{i}\right\}$ of real numbers is monotonic if and only if all the differences $\left(x_{i+1}-x_{i}\right)$ lie (perhaps not strictly) to the same side of 0 on the real line. This motivates:

Definition. A sequence $S=\left\{x_{i}\right\}$ of terms from a finite-dimensional real vector space is monotonic if there is a hyperplane $H$ through the origin such that the differences $d_{i} \equiv x_{i+1}-x_{i}$ all lie in one of the closed half-spaces determined by $H$.

This definition is further justified by:

LEMmA 1. A sequence $S$ of vectors is monotonic if and only if there is a directed line $L$ such that the perpendicular projections of the $x_{i}$ on $L$ form an increasing sequence.

This lemma is easily proved by taking $L$ and $H$ to be perpendicular.

The direction of any such line $L$ is called a direction of monotonicity of $S$. In 1-space there are only two possible directions of monotonicity: increasing and decreasing. In $r$-space the possible directions of monotonicity correspond to the points on the $(r-1)$-sphere.

Any sequence of two real numbers is monotonic. This generalizes to:

LeMma 2. In $r$-space any sequence of $(r+1)$ terms is monotonic.

This can easily be proved geometrically.

Notation. For convenience, let the function $\psi(n)$ which applies in $r$-space be indicated by $\psi_{r}(n)$.

Lemma 3. (a) If $S\left(n^{2}+1\right)=\left\{x_{i}\right\}$ is any sequence of $\left(n^{2}+1\right)$ terms in $r$-space and $L$ is any directed line in $r$-space, then $S$ contains either $a$ subsequence monotonic in the direction of $L$ or a subsequence monotonic in the direction opposite to $L$. (b) $\psi_{r}(n+1) \leqq n^{2}+1$.

Proof. (a) follows easily from Lemma 1 and the Basic Theorem; (b) follows from (a). But (a) is much stronger than (b) because (a) says "for any $L \ldots$ " while (b) says implicitly "there exists an $L$ such that. ..." This suggests that actually $\psi_{r}(n+1)$ is smaller than $\left(n^{2}+1\right)$ in general.

What is the full generalization of the Basic Theorem to $r$-space? In other words, what is the function $\psi_{r}(n)$ ? 
CONJECTURE. $\psi_{r}(n+r)=r n+\left(n^{2}-n+1\right)$.

This conjecture is based solely on the following collection of facts.

BASIC TheOREM. $\psi_{1}(n+1)=n+\left(n^{2}-n+1\right)$.

Lemma 2 (New Form). $\psi_{r}(1+r)=r+(1-1+1)$.

THEOREM 1. $\psi_{r}(2+r) \leqq 2 r+3 \equiv 2 r+(4-2+1)$.

Lemma 4. For $r=1$ and 2 , the $\leqq$ of Theorem 1 becomes $=$.

The proof of Theorem 1 is long and occupies the next section. For $r=1$, Lemma 4 is trivial. To prove Lemma 4 for $r=2$, it is sufficient to exhibit a sequence of 6 vectors from 2-space which contains no monotonic subsequence of 4 terms. That the following is such a sequence may be verified directly:

$$
(2,-1),(3,6),(-3,12),(-3,-12),(3,-6),(2,1) \text {. }
$$

3. Proof of Theorem 1. The basic tool in proving Theorem 1 is

LEMmA 5. If $S(p)=\left\{x_{i}\right\}$ is a sequence in $r$-space, then at least one of the following conditions is true:

(a) $S$ is monotonic;

(b) there exist real numbers $\alpha_{i}>0(i=1, \cdots, p-1)$ such that $\sum \alpha_{i} d_{i}=0$, where $d_{i} \equiv x_{i+1}-x_{i}$.

Proof. It is sufficient to show that the falsity of (b) implies (a). Thus assume that 0 does not belong to the convex cone $C$ $\equiv\left\{\sum \alpha_{i} d_{i} \mid\right.$ all $\left.\alpha_{i}>0\right\}$. Then a well known theorem about convex sets yields that there is a hyperplane $H$ through 0 such that $\bar{C}$ (the topological closure of $C$ ) lies entirely in one of the closed half-spaces determined by $H$. Since $d_{i}$ is in $\bar{C}$ for all $i, S(p)$ is monotonic, and the proof is complete.

Comment. It is possible to modify (b) into a necessary and sufficient condition for non-monotonicity. This condition might be useful in further investigation of the function $\psi_{r}(n)$.

Lemma 6 follows from Lemma 5.

Lemma 6. Let

$$
S(p)=\left\{x_{s}\right\}
$$

be any sequence in r-space, and let

$$
S^{\prime}(q)=\left\{x_{s_{i}}\right\}
$$

be a non-monotonic subsequence of it (of course $1 \leqq s_{1}<s_{2}<\cdots<s_{q}$ $\leqq p)$. Then there exists $a(p-1)$-tuple of real numbers $g=\left\{\gamma_{s}\right\}$ such 
that $\sum \gamma_{s} d_{s}=0$ where $g$ satisfies the following "suitability conditions with respect to $\left(s_{1}, \cdots, s_{q}\right)^{n}$ :

$$
\begin{aligned}
& \left\{\gamma_{1}=\cdots=\gamma_{s_{1}-1}=0,\right. \\
& \left\{\begin{array}{c}
\gamma_{s_{1}}=\cdots=\gamma_{s_{2}-1}>0, \\
\cdot \cdot \cdot \cdot \\
\gamma_{s_{q-1}}=\cdots=\gamma_{s q-1}>0,
\end{array}\right. \\
& \left\{\gamma_{s q}=\cdots=\gamma_{p-1}=0 .\right.
\end{aligned}
$$

Proof. By Lemma 5 there exist strictly positive $\alpha_{i}(i=1, \cdots$, $q-1)$ such that

$$
\sum_{1}^{q-1} \alpha_{i}\left[x_{s i+1}-x_{s_{i}}\right]=0 .
$$

Hence

$$
\sum_{1}^{q-1} \alpha_{i}\left[\sum_{i}^{s_{i}+1^{-1}} d_{j}\right]=0 .
$$

Now define $g=\left\{\gamma_{s}\right\}$ as follows:

$$
\begin{aligned}
& \begin{cases}\gamma_{1}=\cdots=\gamma_{s_{1}-1} & =0,\end{cases} \\
& \begin{cases}\gamma_{s_{1}}=\cdots=\gamma_{s_{2}-1}=\alpha_{1} & >0, \\
\cdot \cdot \cdot \cdot \cdot \cdot \cdot \cdot \cdot \cdot & \cdot \cdot \\
\gamma_{s_{q}}=\cdots=\gamma_{s_{q-1}}=\alpha_{q-1} & >0,\end{cases} \\
& \left\{\gamma_{s_{q}}=\cdots=\gamma_{p-1}=0 .\right.
\end{aligned}
$$

Clearly $\sum \gamma_{s} d_{s}=0$, and the proof is complete.

The structure of the $(p-1)$-tuple $g=\left(\gamma_{1}, \cdots, \gamma_{p-1}\right)$ can be represented by a $q$-block diagram. This is obtained by substituting in $g$ an " $X$ " for each nonzero $\gamma$, and "o" for each zero $\gamma$, and an " $=$ " for each comma between two $\gamma$ 's of one "block" of equal nonzero $\gamma$ 's. A $g$ which is suitable with respect to $(3,5,6,9)$ and which has 10 components is represented by the following 10-dimensional 4-block diagram: (o, o, $X=X, X, X=X=X, 0,0)$. Block diagrams will be used extensively in the following arguments.

At this point it becomes necessary to consider the vectors of the fundamental $r$-space as $r$-tuples of real numbers. We shall write these $r$-tuples vertically and call them column vectors. We adopt the specific notation $d_{s}=$ the column vector $\left(\delta_{s}^{t}\right)$ as $t=1, \cdots, r$, where $d_{s}$ has its usual significance. The sequence $\left\{d_{s}\right\}$, with $s=1$ to 
$p-1$, now becomes a matrix $D=\left\|\delta_{s}^{t}\right\|$ in which $t=1$ to $r$ is the row index and $s=1$ to $p-1$ is the column index. We shall let $d^{t}$ $(t=1, \cdots, r)$ represent the rows of $D$.

We now put Lemma 6 into the proper form for actual use:

LEMMA 7. If $S(p)=\left\{x_{s}\right\}$ is a sequence in $r$-space, which does not contain any $M(q)$, then for each $(p-1)$-dimensional $q$-block diagram there exists $a(p-1)$-dimensional row vector $g$ such that $g$ is perpendicular to all the $d^{t}(t=1, \cdots, r)$ and such that $g$ has the structure of the given $q$-block diagram.

Proof. The $q$-block diagram corresponds to a subsequence $S^{\prime}(q)$ $=\left\{x_{s_{i}}\right\}$ of $S$. Apply Lemma 6 to $S^{\prime}(q)$ and rewrite the equation $\sum \gamma_{s} d_{s}=0$ as $r$ equations $\sum \gamma_{s} \delta_{s}^{t}=0$. These may be written $g \cdot d^{t}=0$ or " $g$ is perpendicular to $d^{t}$." This completes the proof.

The last tool needed to prove Theorem 1 is

Lемма 8. If $S(p)=\left\{x_{s}\right\}$ is not monotonic, the vectors $d^{t}(t=1$ to $r)$ are linearly independent.

Proof. As $S$ is not monotonic, the $d_{s}$ do not all lie on a common hyperplane through the origin, hence span the whole ( $r$-dimensional) space of column vectors. Thus $D$ has column-rank $r$, hence row-rank $r$, which completes the proof.

Theorem 1 is proved indirectly. Assume contrary to the theorem that $S(2 r+3)=\left\{x_{8}\right\}$ contains no $M(r+2)$.

By Lemma 8, the $r(2 r+2)$-dimensional row vectors $d^{t}$ are linearly independent. Now apply Lemma 7 to the following $(r+2)$ different $(2 r+2)$-dimensional $(r+2)$-block diagrams, and label the resulting $g$ 's as shown:

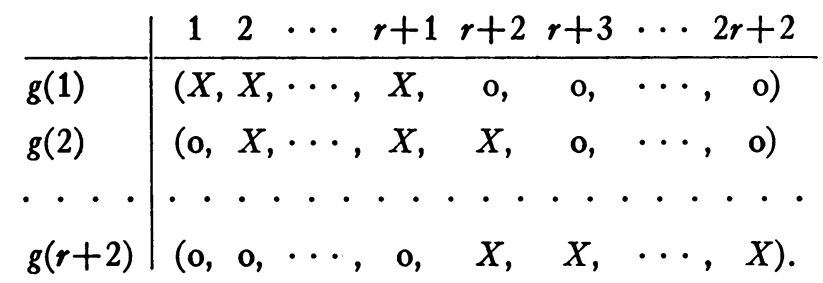

These $g$ 's are called the fundamental $g$ 's. Obviously the $(r+2)$ fundamental $g$ 's are linearly independent. By Lemma 7 every fundamental $g$ is perpendicular to every $d^{t}$. Therefore $[g(1), \cdots, g(r+2)$, $\left.d^{1}, \cdots, d^{r}\right]$ is a basis for the $(2 r+2)$-space of row vectors. From this follows

LEMMA 9. Every $g$ arising from application of Lemma 7 is a linear 
combination of the fundamental $g$ 's.

At this point the proof of Theorem 1 splits into two cases, depending on whether $r$ is odd or even; the former case is simpler and will be considered first.

Assume $r$ is odd. Apply Lemma 7 to the following $(r+1)$ different $(2 r+2)$-dimensional $(r+2)$-block diagrams and label the resulting $g$ 's as shown:

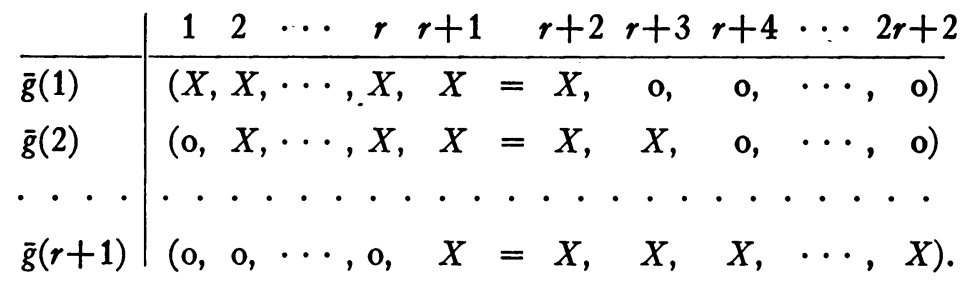

With the aid of Lemma 9 it is easy to see that

$$
\bar{g}(k)=\zeta(k) g(k)+\eta(k) g(k+1)
$$

for properly chosen $\zeta(k)>0$ and $\eta(k)>0$. Introducing an obvious notation for the components of the $g$ 's and the $\bar{g}$ 's, we have

$$
\begin{aligned}
\bar{\gamma}_{r+1}(k) & =\zeta(k) \gamma_{r+1}(k)+\eta(k) \gamma_{r+1}(k+1) \\
= & \bar{\gamma}_{r+2}(k)=\zeta(k) \gamma_{r+2}(k)+\eta(k) \gamma_{r+2}(k+1),
\end{aligned}
$$

which yields that

$$
\left[\gamma_{r+1}(k)-\gamma_{r+2}(k)\right]=-\epsilon(k)\left[\gamma_{r+1}(k+1)-\gamma_{r+2}(k+1)\right]
$$

where $\epsilon(k)$ is a positive constant. Now

$$
\gamma_{r+1}(1)-\gamma_{r+2}(1)>0
$$

because the first term is positive and the second term is 0 . The preceding equation now yields successively

$$
\gamma_{r+1}(2)-\gamma_{r+2}(2)<0, \quad \gamma_{r+1}(3)-\gamma_{r+2}(3)>0,
$$

and so forth. Since $r$ is odd, we obtain

$$
\gamma_{r+1}(r+2)-\gamma_{r+2}(r+2) \equiv-\gamma_{r+2}(r+2)>0
$$

which is false. This completes the proof of Theorem 1 for odd values of $r$.

Now assume that $r$ is even. The following notation is introduced for convenience:

$$
\beta(k) \equiv \gamma_{r+1}(k)-\gamma_{r+2}(k), \quad \beta^{\prime}(k) \equiv \gamma_{r+1}(k)-\gamma_{r+3}(k) .
$$


Using the same method as in the preceding paragraph, the following inequalities are established (but without contradiction here):

$$
\begin{array}{ll}
\beta(k)>0 & \text { if } k \text { is odd; } \\
\beta(k)<0 & \text { if } k \text { is even. }
\end{array}
$$

Now apply Lemma 7 to the following block diagrams and label the resulting $g$ 's as shown:

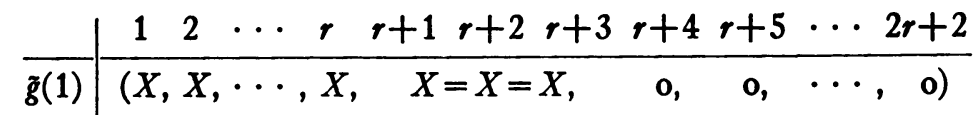

$$
\begin{aligned}
& \tilde{g}(2) \quad(0, X, \cdots, X, \quad X=X=X, \quad X, \quad \text { o, } \cdots, \quad \text { o) } \\
& \tilde{g}(r) \mid(0,0, \cdots, X, \quad X=X=X, \quad X, \quad X, \cdots, X) .
\end{aligned}
$$

With the aid of Lemma 9 and the established inequalities for $\beta(k)$, it is not difficult to show that

$$
\tilde{g}(k)=\zeta(k) g(k)+\eta(k) g(k+1)+\theta(k) g(k+2)
$$

where $\zeta(k), \eta(k)$, and $\theta(k)$ are positive constants. Translating these vector equations into component equations, and using the equalities among the components of the $\tilde{g}$ 's, we have

$$
\begin{aligned}
& \tilde{\gamma}_{r+1}(k)=\zeta(k) \gamma_{r+1}(k)+\eta(k) \gamma_{r+1}(k+1)+\theta(k) \gamma_{r+1}(k+2) \\
= & \tilde{\gamma}_{r+2}(k)=\zeta(k) \gamma_{r+2}(k)+\eta(k) \gamma_{r+2}(k+1)+\theta(k) \gamma_{r+2}(k+2) \\
= & \tilde{\gamma}_{r+3}(k)=\zeta(k) \gamma_{r+3}(k)+\eta(k) \gamma_{r+3}(k+1)+\theta(k) \gamma_{r+3}(k+2) .
\end{aligned}
$$

Subtract the second equation from the first, and then the third from the first:

$$
\begin{aligned}
& 0=\zeta(k) \beta(k)+\eta(k) \beta(k+1)+\theta(k) \beta(k+2), \\
& 0=\zeta(k) \beta^{\prime}(k)+\eta(k) \beta^{\prime}(k+1)+\theta(k) \beta^{\prime}(k+2) .
\end{aligned}
$$

From these equations it follows that

$$
\begin{aligned}
& \zeta(k)=\epsilon(k)\left|\begin{array}{ll}
\beta(k+1) & \beta(k+2) \\
\beta^{\prime}(k+1) & \beta^{\prime}(k+2)
\end{array}\right|, \\
& \eta(k)=\epsilon(k)\left|\begin{array}{ll}
\beta(k+2) & \beta(k) \\
\beta^{\prime}(k+2) & \beta^{\prime}(k)
\end{array}\right|, \\
& \theta(k)=\epsilon(k)\left|\begin{array}{ll}
\beta(k) & \beta(k+1) \\
\beta^{\prime}(k) & \beta^{\prime}(k+1)
\end{array}\right|
\end{aligned}
$$

where $\epsilon(k)$ is a properly chosen constant of proportionality. 
Call the three determinants $\mathrm{Z}(k), \mathrm{H}(k)$, and $\Theta(k)$ respectively. Since $\zeta(k), \eta(k)$, and $\theta(k)$ are all positive, $\mathrm{Z}(k), \mathrm{H}(k)$, and $\Theta(k)$ must all have the same sign as $\epsilon(k)$. Furthermore, as $\mathrm{Z}(k) \equiv \Theta(k+1)$, all the determinants have the same sign for all $k$ (from 1 to $r$ ). To evaluate the sign of $\Theta(1)$ we use the already established inequalities for the $\beta$ 's and find the sign of the $\beta^{\prime \prime}$ 's from direct examination of the block diagrams of the $g$ 's. We see that

$$
\Theta(1)=\left|\begin{array}{ll}
+ & - \\
+ & +
\end{array}\right|>0,
$$

so that all the determinants are positive.

Similarly, we find that

$$
\mathrm{z}(1)=\left|\begin{array}{cc}
- & + \\
+ & ?
\end{array}\right|
$$

For this to be positive, "?" must be " - ", so that $\beta^{\prime}(3)<0$. Using this result we see that

$$
\mathrm{Z}(2)=\left|\begin{array}{cc}
+ & - \\
- & ?
\end{array}\right|
$$

For this to be positive, "?" must be " + ," so that $\beta^{\prime}(4)>0$. Similarly, $\beta^{\prime}(5)<0, \beta^{\prime}(6)>0$, and so forth. Since $r$ is even, $\beta^{\prime}(r+2)>0$; however, direct examination of the block diagram shows that $\beta^{\prime}(r+2)<0$. This contradiction completes the proof of Theorem 1 for even values of $r$, and hence the whole proof.

4. Second generalization-relation spaces. In the following sections we again generalize the Basic Theorem, but in a manner quite different from that of the preceding sections.

The Basic Theorem is not in essence a statement about the real number system. To see this, consider any set $X$ with an arbitrary binary relation $C$ over it. (No assumptions are made about $C$; for example, it need not be transitive.) Let us say that $S=\left\{x_{i}\right\}$ is $C$ monotonic $\left(\varangle\right.$-monotonic) if $x_{i} \subset x_{i+1}\left(x_{i} \varangle x_{i+1}\right)$ for all $i$. Call $S$ monotonic if it is either $\subset$-monotonic or $\varangle$-monotonic. Then for sequences over $X$ it is still true that $\psi(n+1) \leqq n^{2}+1$, and for a "general" space $X$ it is true that $\psi(n+1)=n^{2}+1$. The inequality may be proved exactly as before.

What is the meaning of the " 2 " in $\left(n^{2}+1\right)$ ? The answer simple: it is the number of relations $(C$ and $\Phi$ ) of which at least one must hold between any two elements. The " 2 " is generalized to a " $k$ " in Theorem 2. 
Definition. A $k$-relation space ( $k R$-space for short) consists of a set $X$ and $k$ binary relations $C_{h}$ over $X(h=1, \cdots, k)$ satisfying one axiom: for every $x, y$ in $X$, there is at least one $h$ depending on $x$ and $y$ such that $x \subset_{h} y$.

Definitron. A sequence $S=\left\{x_{i}\right\}$ is $C_{h}$-monotonic if $x_{i} \subset_{h} x_{i+1}$ for all $i$.

Definition. A sequence $S$ is monotonic if there is at least one $h$ for which it is $C_{h}$-monotonic.

Extended BAsic Theorem. For sequences over a 2-relation space, $\psi(n+1) \leqq n^{2}+1$. Furthermore, there are 2-relation spaces for which $\psi(n+1)=n^{2}+1$.

TheOREM 2. For sequences over a $k R$-space, $\psi(n+1) \leqq n^{k}+1$. Furthermore, there are $k R$-spaces for which $\psi(n+1)=n^{k}+1$.

In the $k R$-space to be described $\psi(n+1)=n^{k}+1$. Let $X$ consist of all real polynomials in the variable $\xi$ of degree $\leqq k-1$. The relations $C_{h}$ are defined by

$$
p(\xi) \subset_{h} q(\xi) \quad \text { if }[p(\xi)-q(\xi)] \text { has exactly degree }(k-h) .
$$

(The zero polynomial is assigned degree 0.) It is trivial to show that this is a $k R$-space, and the following $S\left(n^{k}\right)$ contains no $M(n+1)$ :

$$
\begin{gathered}
\xi^{k-1}+\xi^{k-2}+\cdots+\xi+1, \\
\xi^{k-1}+\xi^{k-2}+\cdots+\xi+2, \\
\cdot \cdot \cdot \cdot \cdot \cdot \cdot \cdot \cdot \cdot, \\
\xi^{k-1}+\xi^{k-2}+\cdots+\xi+n, \\
\xi^{k-1}+\xi^{k-2}+\cdots+2 \xi+1, \\
\cdot \cdot \cdot \cdot \cdot \cdot \cdot \cdot \cdot \cdot \cdot, \\
\xi^{k-1}+\xi^{k-2}+\cdots+2 \xi+n, \\
\cdot \cdot \cdot \cdot \cdot \cdot \cdot \cdot \cdot \cdot \cdot, \\
\cdot \cdot \cdot \cdot \cdot \cdot \cdot \cdot \cdot \cdot \cdot, \\
n \xi^{k-1}+n \xi^{k-2}+\cdots+n \xi+n .
\end{gathered}
$$

The proof ${ }^{2}$ that $\psi(n+1) \leqq n^{k}+1$ in a $k$-relation space rests on Lemma 10 which (for real numbers) is stated in the paper by Erdös and Szekeres.

2 For the basic idea of this proof I am indebted to the referee, who suggested a proof far simpler than the one originally contained in my paper. However, by using Lemma 10, not originally in my paper and not known to the referee, I have further simplified his proof. 
Lemмa 10. If $\left(X, C_{1}, C_{2}\right)$ is a 2-relation space, then any sequence $S(p q+1)$ either contains a $C_{1}$-monotonic subsequence $M(p+1)$ or $a$ $C_{2}$-monotonic subsequence $M(q+1)$.

This lemma may easily be proved in the same way as the Extended Basic Theorem.

Now we proceed by an induction on $k$. If $\left(X, \subset_{1}, \cdots, \subset_{k+1}\right)$ is a $(k+1)$-relation space, and $S\left(n^{k+1}+1\right)$ is a sequence over it, define $\ll_{1}$ and $\ll_{2}$ by

$$
\begin{array}{ll}
x \ll_{1} y, & \text { if } x \subset_{h} y \text { for any } h \text { from } 1 \text { to } k, \\
x \ll_{2} y & \text { if } x \subset_{k+1} y .
\end{array}
$$

Now $\left(X, \ll_{1}, \ll_{2}\right)$ is a 2 -relation space. Hence by Lemma $10, S$ contains either $M_{1}\left(n^{k}+1\right)$ which is $\ll_{1}$-monotonic or $M_{2}(n+1)$ which is $\ll_{2}$-monotonic. In the latter case the proof is complete as $M_{2}(n+1)$ is also $C_{k+1}$-monotonic. In the former case, let $M_{1}$ denote the set of elements in $M_{1}\left(n^{k}+1\right)$ and define $C^{h}$ over $M_{1}$ by

$$
x \subset^{h} y \quad \text { if } x \subset_{h} y \text { or if } y \text { precedes } x \text { in } M_{1}\left(n^{k}+1\right) .
$$

Then $\left(M_{1}, \subset^{1}, \cdots, \subset^{k}\right)$ is a $k R$-space. Hence by the induction hypothesis $M_{1}\left(n^{k}+1\right)$ must contain an $M(n+1)$ which is $C^{h}$-monotonic for some $h$ from 1 to $k$. But then $M(n+1)$ is $C_{h}$-monotonic, which completes the proof.

5. De Bruijn's Theorem-a generalization. In some unpublished work N. G. de Bruijn has generalized the Basic Theorem to sequences of $m$-tuples of real numbers. He defines such a sequence to be monotonic if each component sequence is monotonic. (Thus there are $2^{m}$ "directions" of monotonicity.)

De BruijN's Theorem. Over the space of $m$-tuples, $\psi(n+1)=n^{2^{m}}+1$.

His proof is simply an $m$-fold application of the Basic Theorem. From $S\left(n^{2^{m}}+1\right)$ pick a subsequence $S_{1}\left(n^{2^{m-1}}+1\right)$ whose first components are monotonic. From $S_{1}$ pick a subsequence $S_{2}\left(n^{2^{m-2}}+1\right)$ whose second components are monotonic; and so forth. This eventually yields $S_{m}(n+1)$ which is monotonic. This shows that $\psi(n+1)$ $\leqq n^{2^{m}}+1$; the opposite inequality is easily verified.

De Bruijn's Theorem inspires Theorem 3, which is at once a generalization of De Bruijn's Theorem and of Theorem 2.

Definition. A joint relation-space with coefficients $k_{1}, \cdots, k_{m}$ consists of $m$ different relation-spaces over the same set $X$ such that the $l$ th space is a $k_{l} R$-space. 
The relations are denoted by $C_{h}^{l}$, where $h=1, \cdots, k_{l}$ and $l=1, \cdots, m$.

Definition. A sequence is monotonic over a joint relation-space if it is simultaneously monotonic over every one of the $k_{l} R$-spaces.

TheOREM 3. Over a joint relation-space, $\psi(n+1) \leqq n^{k_{1} k_{2} \cdots k_{m}}+1$.

De Bruijn's Theorem is a special case of Theorem 3 in which all the coefficients are 2 and the set $X$ consists of the real $m$-tuples. However his proof cannot be extended to prove Theorem 3 , for his proof depends on the transitivity of his relations which is not assumed in Theorem 3.

However Theorem 3 may be proved as a trivial corollary to Theorem 2. Simply define $k_{1} k_{2} \cdots k_{m}$ new relations over $X$ by

$$
x \ll_{h_{1}}, \ldots, h_{m} y \text { only if } x C_{h_{l}}^{l} y \text { for all } l \text {. }
$$

Then $X$ and the new relations form a $k_{1} k_{2} \cdots k_{m} R$-space. Use of Theorem 2 then completes the proof.

Princeton University 